\title{
Existence, Multiplicity, and Stability of Positive Solutions of a Predator-Prey Model with Dinosaur Functional Response
}

\author{
Xiaozhou Feng, ${ }^{1}$ Kenan Shi, ${ }^{1}$ Jianhui Tian, ${ }^{2}$ and Tongqian Zhang,4 \\ ${ }^{1}$ Department of Mathematics and Physics, Xian Technological University, Xi'an 710032, China \\ ${ }^{2}$ School of Mechanical Engineering, Xian Technological University, Xian 710032, China \\ ${ }^{3}$ College of Mathematics and Systems Science, Shandong University of Science and Technology, Qingdao 266590, China \\ ${ }^{4}$ State Key Laboratory of Mining Disaster Prevention and Control Co-Founded by Shandong Province and \\ the Ministry of Science and Technology, Shandong University of Science and Technology, Qingdao 266590, China
}

Correspondence should be addressed to Xiaozhou Feng; flxzfxz8@163.com

Received 19 April 2017; Accepted 10 July 2017; Published 13 August 2017

Academic Editor: Sebastian Anita

Copyright (C) 2017 Xiaozhou Feng et al. This is an open access article distributed under the Creative Commons Attribution License, which permits unrestricted use, distribution, and reproduction in any medium, provided the original work is properly cited.

We investigate the property of positive solutions of a predator-prey model with Dinosaur functional response under Dirichlet boundary conditions. Firstly, using the comparison principle and fixed point index theory, the sufficient conditions and necessary conditions on coexistence of positive solutions of a predator-prey model with Dinosaur functional response are established. Secondly, by virtue of bifurcation theory, perturbation theory of eigenvalues, and the fixed point index theory, we establish the bifurcation of positive solutions of the model and obtain the stability and multiplicity of the positive solution under certain conditions. Furthermore, the local uniqueness result is studied when $b$ and $d$ are small enough. Finally, we investigate the multiplicity, uniqueness, and stability of positive solutions when $k>0$ is sufficiently large.

\section{Introduction}

The dynamic relationship between predator and their prey is one of dominant themes in ecology and mathematical ecology. Some models have been studied from various views and obtained many good results (see [1-16] and the references therein). In this paper, we are concerned with the predatorprey model with Dinosaur functional response under the homogeneous Dirichlet boundary conditions as follows:

$$
\begin{aligned}
\frac{\partial u}{\partial t}-\Delta u & =u\left(a-u-b v e^{-k u}\right), \quad x \in \Omega, \\
\frac{\partial v}{\partial t}-\Delta v & =v\left(c-v+d u e^{-k u}\right), \quad x \in \Omega, \\
u & =v=0, \quad x \in \partial \Omega \\
u(x, 0) & =u_{0}(x), \\
v(x, 0) & =v_{0}(x),
\end{aligned}
$$

where $\Omega$ is a bounded domain in $R^{n}(n \geq 1)$ with smooth boundary $\partial \Omega$ and $u, v$ stand for the densities of prey and predators, respectively. $a, b, c, d, k$ are positive constants. $a$ and $c$ denote prey intrinsic growth rate and predator intrinsic growth rate and then decrease to zero when $u \rightarrow \infty$, as follows:

$$
\begin{aligned}
& \frac{d}{d u} u e^{-k u}>0, \quad 0 \leq u<\frac{1}{k}, \\
& \frac{d}{d u} u e^{-k u}<0, \quad u>\frac{1}{k} .
\end{aligned}
$$

The Dinosaur reaction term is an improvement or simplification of the Ivlev-type reaction term, and the change on the species density of prey better than Ivlev-type functional response $h\left(1-e^{-k u}\right)$ can be explained. It is easy to see that the Dinosaur reaction term describes prey focus on the fight against predators when the species density of prey is large enough, so as to achieve better defense or disguise itself. To see more biological significance of systems with Ivlev-type functional responses, one can resort to [17-28] and their 
contents. The research on existence and uniqueness of the limit cycle of a predator-prey model with Ivlev response can be found in $[22,23]$. The permanence and existence and stability of positive periodic solutions of the model were studied in [24-26]. The spatial pattern formation of the model was investigated by using Hopf bifurcation in [27, 28]. Some dynamical behavior analysis of the Ivlev response predator-prey systems was established in [17-21]. However, the researches on system (1) are very few. Hence, this paper mainly aims at establishing the existence, bifurcation, and multiplicity of positive solutions on the corresponding elliptic equations to system (1).

The research on the steady-states in reaction-diffusion model is the hot point question $[1-4,9,10,13,16]$. In the present paper, we study the steady-state problem corresponding to (1), with the specific form as follows:

$$
\begin{aligned}
-\Delta u & =u\left(a-u-b v e^{-k u}\right), \quad x \in \Omega, \\
-\Delta v & =v\left(c-v+d u e^{-k u}\right), \quad x \in \Omega, \\
u & =v=0, \quad x \in \partial \Omega .
\end{aligned}
$$

Motivated by the papers [1-4], in the present paper, we mainly consider the positive solution of (3). In Section 2, we give some basic results and calculate the fixed point index by the fixed point index theory. In Section 3, we apply the results obtained in Section 2 to study the existence of positive solutions. In Section 4, by virtue of bifurcation theory, perturbation theory of eigenvalues, and the fixed point index theory, we establish the bifurcation of positive solutions of (3) and obtain the stability and multiplicity of the positive solution under certain conditions. Furthermore, the local uniqueness result is studied when $b$ and $d$ are small enough. Finally, we investigate the multiplicity, uniqueness, and stability of positive solutions when $k>0$ is sufficiently large.

\section{Preliminaries}

In this section, we mainly calculate the fixed point index by the fixed point index theory, in the following, and set up some definitions which are used in last paper.

Let $E$ be a Banach space. $W \subset E$ is called a wedge if $W$ is a closed convex set $\alpha W \subset W$ for all $\alpha \geq 0$. For any $y \in W$, define

$$
\begin{aligned}
& W_{y}=\{x \in E: \exists r=r(x)>0, \text { s.t. } y+r s \in W\}, \\
& S_{y}=\left\{x \in \bar{W}_{y}:-x \in \bar{W}_{y}\right\},
\end{aligned}
$$

and we always assume that $E=\overline{W-W}$. Let $T: W_{y} \rightarrow W_{y}$ be a compact linear operator on $E$. We say that $T$ has property $\alpha$ on $\bar{W}_{y}$ if there exist $t \in(0,1)$ and $\omega \in \bar{W}_{y} \backslash S_{y}$ such that $\omega-t T \omega \in S_{y}$. Suppose that $F: W \rightarrow W$ is a compact operator for the fixed point $y_{0} \in W$, and $F$ is Fréchet differentiable at $y_{0}$. Set $L=F^{\prime}\left(y_{0}\right)$ as the Fréchet differentiable of $F$ at $y$; hence $L: \bar{W} \rightarrow \bar{W}$.
Lemma 1 (see $[29,30]$ ). Assuming that $I-L$ is invertible on $E$, then one has

(i) L has property $\alpha$ on $\bar{W}$, and then inde $x_{w}\left(F, y_{0}\right)=0$.

(ii) $L$ does not have property $\alpha$ on $\bar{W}$, and then index ${ }_{w}\left(F, y_{0}\right)=(-1)^{\sigma}$, where $\sigma$ is the sum of algebra multiplicities of the eigenvalues of $L$ which are greater than one.

Let $q(x) \in L^{\infty}, k>0$ denote the principal eigenvalue of the following problem:

$$
-\Delta(\phi)+q(x) \phi=\lambda \phi, \quad x \in \Omega, \phi=0, x \in \partial \Omega,
$$

and, by $\lambda_{1}(q(x))$, it follows from $[3,4]$ that $\lambda_{1}(q(x))$ is strictly increasing in the case that $q_{1}(x) \leq q_{2}(x)$ and $q_{1}(x) \neq q_{2}(x)$ implies $\lambda_{1}\left(q_{1}(x)\right)<\lambda_{1}\left(q_{2}(x)\right)$. For convenience we denote $\lambda_{1}(0)$ by $\lambda_{1}$. Let $r(T)$ be the spectral radius of linear operator $T$ on Banach space.

Lemma 2 (see $[24,30])$. Suppose that $q(x) \in C^{1}$, and $P$ is large enough such that $\forall x \in \Omega,-q(x)+P>0$. Then the following results hold:

(i) $\lambda_{1}\left(q_{1}(x)\right)<0 \Rightarrow r\left[(-\Delta+P)^{-1}(-q(x)+P)\right]>1$.

(ii) $\lambda_{1}\left(q_{1}(x)\right)>0 \Rightarrow r\left[(-\Delta+P)^{-1}(-q(x)+P)\right]<1$.

(iii) $\lambda_{1}\left(q_{1}(x)\right)=0 \Rightarrow r\left[(-\Delta+P)^{-1}(-q(x)+P)\right]=1$.

Next, we present some a priori estimate by maximum principle which is similar to $[3,4]$; its proof will be omitted.

Theorem 3. Any positive solution $(u, v)$ of (3) has an a priori boundary; that is,

$$
\begin{aligned}
& u(x) \leq a, \\
& v(x) \leq c+\frac{d}{k e} .
\end{aligned}
$$

Consider the following equation:

$$
-\Delta \theta=\theta(\rho-\theta), \quad x \in \Omega, \theta=0, x \in \partial \Omega
$$

it is easy to see that (7) has unique positive solution denoted by $\theta_{\rho}$ when $\rho>\lambda_{1, k}$. Hence, if $a>\lambda_{1}$, then (3) has a semitrivial solution $\left(\theta_{a}, 0\right)$; if $c>\lambda_{1}$, (3) has a semitrivial solution $\left(0, \theta_{c}\right)$.

Now, some notations are introduced as follows:

(1) $E: c(\bar{\Omega}) \oplus c(\bar{\Omega})$, here $c(\bar{\Omega}):=\{\phi \in c(\bar{\Omega}), x \in \partial \Omega, \quad v=$ $0\}$.

(2) $W: K \oplus K$, here $K:=\{\phi \in C(\bar{\Omega}: 0 \leq \phi(x), x \in \Omega)\}$.

(3) $D:\{(u, v) \in E: u \leq a+1, v \leq v+d / k e+1\}$.

(4) $D^{\prime}:=\operatorname{int} D \cap W$. 

form:

Define a compact operator $F: D^{\prime} \rightarrow W$ by the following

$$
F(u, v)=(-\Delta+P)^{-1}\left(\begin{array}{l}
u\left(a-u-b v e^{-k u}\right)+P u \\
v\left(c-v+d u e^{-k u}\right)+P u
\end{array}\right),
$$

where $P$ is sufficiently large constant such that $P>\max \{a$ $+b(c+d / k e), c+d / k e\}$. It follows that (3) has a positive solution in $W$ if and only if $F$ has a positive fixed point in $D^{\prime}$. Hence, $(0,0),\left(\theta_{a}, 0\right),\left(0, \theta_{c}\right)$ are the only isolated fixed points of $F$ if $a, c>\lambda_{1}$. Therefore, $\operatorname{index}_{w}(F$, $(0,0)), \operatorname{index}_{w}\left(F,\left(\theta_{a}, 0\right)\right)$, index $\left(F,\left(0, \theta_{c}\right)\right)$ are well-defined, and the indices can be calculated, similar to Lemmas 2.3-2.4 in [3]; we can directly give Lemmas 4 and 5 as follows.

Lemma 4. Suppose $a>\lambda_{1}$.

(i) $\operatorname{index}_{w}\left(F, D^{\prime}\right)=1$.

(ii) index $_{w}(F,(0,0))=0$.

(iii) If $c>\lambda_{1}\left(-d \theta_{a} e^{-k \theta_{a}}\right)$, then index $\left(F,\left(\theta_{a}, 0\right)\right)=0$.

(iv) If $c<\lambda_{1}\left(-d \theta_{a} e^{-k \theta_{a}}\right)$, then index $\left(F,\left(\theta_{a}, 0\right)\right)=1$.

Lemma 5. Suppose $c>\lambda_{1}$. (i) If $a>\lambda_{1}\left(b \theta_{c}\right)$, then index $_{w}\left(F,\left(0, \theta_{c}\right)\right)=0$; (ii) if $a<\lambda_{1}\left(b \theta_{c}\right)$, then index ${ }_{w}(F,(0$, $\left.\left.\theta_{c}\right)\right)=1$.

\section{Existence of Positive Solutions}

In the section, we apply the result of Lemmas 4 and 5 obtained in Section 2 to study the existence and nonexistence of positive solutions of (3).

Theorem 6. (i) If $c>\lambda_{1}, a>\lambda_{1}\left(b \theta_{c}\right)$, then (3) has at least one positive solution.

(ii) If $c<\lambda_{1}$, then (3) has a positive solution if and only if $a>\lambda_{1}$ and $c>\lambda_{1}\left(-d \theta_{a} e^{-k \theta_{a}}\right)$.

Proof. (i) According to Lemmas 4 and 5 and the additivity property of the index, we have

$$
\begin{aligned}
\operatorname{index}_{w} & \left(F, D^{\prime}\right)-\operatorname{index}_{w}(F,(0,0)) \\
& -\operatorname{index}_{w}\left(F,\left(\theta_{a}, 0\right)\right)-\operatorname{index}_{w}\left(F,\left(0, \theta_{c}\right)\right)=1 .
\end{aligned}
$$

Hence, (3) has at least one positive solution.

(ii) If $c<\lambda_{1}$, then (3) has no solution taking the form $(0, v)(v>0)$. Supposing $a>\lambda_{1}$ and $c>\lambda_{1}\left(-d \theta_{a} e^{-k \theta_{a}}\right)$, note that $c<\lambda_{1}$, and by Lemma 5 , we can get the following:

$$
\begin{gathered}
\operatorname{index}_{w}\left(F, D^{\prime}\right)-\operatorname{index}_{w}(F,(0,0)) \\
-\operatorname{index}_{w}\left(F,\left(\theta_{a}, 0\right)\right)=1 .
\end{gathered}
$$

Hence, (3) has at least one positive solution. So the sufficiency is proved. Conversely, suppose that $(u, v)$ is a positive solution of (3); then $a<\lambda_{1}, u<\theta_{a}$. This is because $(u, v)$ satisfies

$$
-\Delta v=v\left(c-v+d u e^{-k u}\right), \quad x \in \Omega, u=0, x \in \partial \Omega .
$$

It follows that $0=\lambda_{1}\left(-c+v-d u e^{-k u}\right)>\lambda_{1}\left(-c-d \theta_{a} e^{-k \theta_{a}}\right)$. This completes the proof of Theorem 6 .
Theorem 7. (i) If $a \leq \lambda_{1}$, then (3) has no positive solutions; if $a \leq \lambda_{1}$ and $c \leq \lambda_{1}$, then (3) has no nonnegative nonzero solution.

(ii) If $c \leq \lambda_{1}$, then the conditions $a>\lambda_{1}, c+d / k e>\lambda_{1}$ are necessary for the existence of positive solution of (3). In addition, as a $<1 / k$, the condition $c>\lambda_{1}\left(-d \theta_{a} e^{-k \theta_{a}}\right)$ can take place of the necessary condition $c+d / k e>\lambda_{1}$.

(iii) If $c>\lambda_{1}$, then the conditions $a>\max \left\{\lambda_{1}\left(b \theta_{c}\right)\right.$, $\lambda_{1}\left(b \theta_{c}\right) e^{-k \theta_{a}}$ are necessary for the existence of positive solutions of (3).

Proof. (i) Suppose that $(u, v)$ is a positive solution of (3); then $(u, v)$ satisfies

$$
-\Delta u=u\left(a-u-b v e^{-k u}\right) \leq a(a-u),
$$

$$
x \in \Omega, u=0, x \in \partial \Omega,
$$

so we have $a=\lambda_{1}\left(u+b v e^{-k u}\right)$. According to the comparison principle of eigenvalues, we have $a>\lambda_{1}$, a contradiction. Next, we suppose that $(u, v)$ is a nonnegative nonzero solution of (3). If $u \neq \equiv 0, v \equiv 0$, then $a>\lambda_{1}$, a contradiction to the previous proof. Similarly, it is easy to see that $c>\lambda_{1}$, when $u \equiv 0, v \neq 0$, which derives a contradiction.

(ii) Suppose that $(u, v)$ is a positive solution of (3); it follows that $a>\lambda_{1}$, and then the positive semitrivial solution $\theta_{a}$ exists. Thanks to

$$
\begin{aligned}
-\Delta u=u\left(a-u-b v e^{-k u}\right) \leq & a(a-u), \\
& x \in \Omega, u=0, x \in \partial \Omega,
\end{aligned}
$$

$u$ is a lower solution of (3), and due to the uniqueness of $\theta_{a}$, $u<\theta_{a}$. Thus, since $v$ satisfies the problem

$$
-\Delta v=v\left(c-v+d u e^{-k u}\right), \quad x \in \Omega, u=0, x \in \partial \Omega
$$

it follows from Lemma 2(iii) that

$$
0=\lambda_{1}\left(-c+v-d u e^{-k u}\right)>\lambda_{1}\left(-c-d \theta_{a} e^{-k \theta_{a}}\right) .
$$

Moreover, as $a<1 / k$, due to $u<\theta_{a} \leq a<1 / k$, by the second equation of (3), we get $c>\lambda_{1}\left(-d \theta_{a} e^{-k \theta_{a}}\right)$.

(iii) Supposing that $(u, v)$ is a positive solution of (3), by the proof of (ii), $\theta_{a}$ exists and $u \leq \theta_{a}$. Similarly, the given condition $c>\lambda_{1}$ can deduce the existence of the positive $\theta_{c}$ of (3), $\theta_{c} \leq v$. Similar to the proof of (i), we also obtain the fact that $a=\lambda_{1}\left(u+b v e^{-k u}\right)$ has the minimum at $u=0, v=\theta_{c}$ or $u=\theta_{a}, v=\theta_{c}$, which derives the desired result.

This completes the proof of Theorem 7.

Theorem 8. If one of the following conditions holds, then (3) has no positive solutions:

(i) $b \geq e^{k a}$ and $a \leq c$.

(ii) $b<e^{k a}$ and $a+d / k e \leq b e^{k a}(c+d / k e)$. 
Proof. (i) Assume that there is a positive solution $(u, v)$ of (3). Recalling the results of Theorem 3 , as $b \geq e^{k a}$ and $a \leq c$, we get

$$
\begin{aligned}
0 & =\lambda_{1}\left(-a+u+b v e^{-k u}\right) \\
& \geq \lambda_{1}\left(-c+v-d u e^{-k u}-v+b v e^{-k u}+u+d u e^{-k u}\right) \\
& \geq \lambda_{1}\left[-c+v-d u e^{-k u}-\left(1-b e^{-k u}\right) v\right] \\
& \geq \lambda_{1}\left[-c+v-d u e^{-k u}\right]
\end{aligned}
$$

which is a contradiction to Lemma 2(iii).

(ii) Assume that there is a positive solution $(u, v)$ of (3). By Theorem 3, if $b<e^{k a}$ and $a+d / k e \leq b e^{k a}(c+d / k e)$, we have

$$
\begin{aligned}
0= & \lambda_{1}\left(-a+u-b v e^{-k u}\right) \geq \lambda_{1}\left(-c+v-d u e^{-k u}-a\right. \\
& \left.+c-v+u+b v e^{-k u}+d u e^{-k u}\right) \geq \lambda_{1}[-c+v \\
& \left.-d u e^{-k u}-a+c-\left(c+\frac{d}{k e}\right)\left(1-b e^{-k a}\right)\right] \\
& \geq \lambda_{1}\left[-c+v-d u e^{-k u}\right]
\end{aligned}
$$

which is a contradiction to Lemma 2(iii).

This completes the proof of Theorem 8 .

\section{Bifurcation and Multiplicity}

In this section, taking $a$ as a main bifurcation parameter, we shall prove that (3) has at least two positive solutions when the parameters involved in (3) satisfy some ranges. In particular, the uniqueness of positive solutions is established when $b$ and $d$ are small enough. By the local bifurcation theory $[6,31]$ or Section 13 in [30], the branch of positive solutions of (3) bifurcates from $\left(0, \theta_{c}, a\right)$ (or $\left.\left(\theta_{a}, 0, c\right)\right)$ when $c>\lambda_{1}$ (or $a>$ $\left.\lambda_{1}\right)$ is established. Now we give the following Lemma where the proof is similar to Theorem 3.8 in [3].

Lemma 9. (i) If $c>\lambda_{1}$ and $a \geq \lambda_{1}\left(b \theta_{c}\right)$, then (3) has a positive solution which bifurcates from $\left(0, \theta_{c}, a\right)$ if and only if $a=\tilde{a}$, and here $\tilde{a}:=\lambda_{1}\left(b \theta_{c}\right)$.

(ii) If $a>\lambda_{1}$ and $\lambda_{1}\left(-d \theta_{a} e^{-k \theta_{a}}\right) \leq c \leq \lambda_{1}$, then (3) has a positive solution which bifurcates from $\left(\theta_{a}, 0, c\right)$ if and only if $c=\widetilde{c}$, and here $\widetilde{c}:=\lambda_{1}\left(-d \theta_{a} e^{-k \theta_{a}}\right)$.

More precisely, all positive solutions near $\tilde{a}:=\lambda_{1}\left(b \theta_{c}\right)$ are defined by

$$
\begin{gathered}
\left\{(u(s), v(s), a(s))=s \Phi+O\left(s^{2}\right), \theta_{c}+s \Psi\right. \\
\left.\quad+O\left(s^{2}\right), \widetilde{a}+a_{1} s+O\left(s^{2}\right): 0<s<\sigma\right\},
\end{gathered}
$$

where $\Phi$ is the principal eigenfunction of $\tilde{a}$, and it follows from the following problem:

$$
\begin{aligned}
-\Delta \xi+\left(\tilde{a}-b \theta_{c}\right) \xi & =0, \quad x \in \Omega, \\
-\Delta \eta+\left(c-2 \theta_{c}\right) \eta & =-d \theta_{c} \xi, \quad x \in \Omega, \\
\xi & =\eta=0, \quad x \in \partial \Omega,
\end{aligned}
$$

where $\Psi=\left(-\Delta+c-2 \theta_{c}\right)^{-1}\left(d \theta_{c} \Phi\right)$.

Remark 10. In Section 3, by Theorems 6 and 7, we can know the sufficient condition and necessary condition on the existence of positive solutions and find that there exists a gap between $a>\lambda_{1}\left(b \theta_{c}\right)$ and $a>\lambda_{1}\left(b \theta_{c}\right) e^{-k \theta_{a}}$.

Next, using the constant $a$ as a main bifurcation parameter, we get the following theorem which establishes the multiplicity and stability results of positive solution for (3) in the gap.

Theorem 11. Suppose $c>\lambda_{1}$ and $\int_{\Omega} \Phi^{3}\left(1-b k \theta_{c}\right)<0$. There exists small enough $\varepsilon>0$ such that the local bifurcation of the positive solution $(u(s), v(s))$ bifurcates from $\left(0, \theta_{c}, \widetilde{a}\right)$ which is nondegenerate and unstable for $a \in(\widetilde{a}-\varepsilon, \widetilde{a})$ and $0<d \ll 1$. In addition, (3) has at least two positive solutions.

Proof. Firstly, we prove that any positive solutions bifurcated from $\left(0, \theta_{c}\right)$ are nondegenerate and unstable. To complete this, we need only show that there exists a sufficiently small $\varepsilon>0$ such that, for $a \in(\widetilde{a}-\varepsilon, \widetilde{a})$, any positive solution $(u(s), v(s))$ of $(3)$ is nondegenerate and the linearized eigenvalue problem

$$
\begin{aligned}
& -\Delta \xi-\xi\left(a(s)-2 u(s)+b v(s) e^{-k u(s)}\right. \\
& \left.+b u(s) v(s) k e^{-k u(s)}\right)+b u(s) e^{-k u(s)} \eta=\mu \xi, \\
& x \in \Omega, \\
& -\Delta \eta-\xi\left(d v(s) e^{-k u(s)}-d u(s) v(s) k e^{-k u(s)}\right)-(c \\
& \left.-2 v(s)+d u(s) e^{-k u(s)}\right) \eta=\mu \eta, \quad x \in \Omega
\end{aligned}
$$

has a unique eigenvalue $\widehat{\mu}$ such that $\operatorname{Re}(\widehat{\mu})<0$ with multiplicity one. Suppose that $\left\{\varepsilon_{n}>0\right\},\left\{d_{n}\right\},\left\{s_{n}>0\right\}$ are the sequences which converge to 0 as $n \rightarrow \infty$. Thanks to $a=$ $\tilde{a}+a_{1} s+O\left(s^{2}\right)$, there exists the sequence $\left\{a_{n}\right\}$ such that $a_{n} \in$ $\left(\widetilde{a}-\varepsilon_{n}, \widetilde{a}\right)$ as $n \rightarrow \infty$. Set $\left(u_{n}, v_{n}\right)$ as a solution of (3). So linearized problem (20) can be denoted by

$$
\begin{aligned}
&- \Delta \xi_{n}-\xi_{n}\left(a_{n}-u_{n}-b v_{n} e^{-k u_{n}}+b u_{n} v_{n} k e^{-k u_{n}}\right) \\
&+b u_{n} e^{-k u_{n}} \eta_{n}=\mu_{n} \xi_{n}, \quad x \in \Omega, \\
&- \Delta \eta_{n}-\xi_{n}\left(d_{n} v_{n} e^{-k u_{n}}-d_{n} u_{n} v_{n} k e^{-k u_{n}}\right) \\
&-\left(c-2 v_{n}+d_{n} u_{n} k e^{-k u_{n}}\right) \eta_{n}=\mu_{n} \eta_{n}, \quad x \in \Omega, \\
& \xi_{n}=\eta_{n}=0, \quad x \in \partial \Omega .
\end{aligned}
$$


Notice that, as $n \rightarrow \infty$, problem (21) converges to the following problem:

$$
\begin{aligned}
\Delta \xi-\left(\widetilde{a}-b \theta_{c}\right) \xi & =\mu \xi, \quad x \in \Omega, \\
-\Delta \eta-\left(c-2 \theta_{c}\right) \eta & =\mu \eta, \quad x \in \Omega, \\
\xi & =\eta=0, \quad x \in \partial \Omega,
\end{aligned}
$$

and since $c=\lambda_{1}\left(\theta_{c}\right)<\lambda_{1}\left(2 \theta_{c}\right)$, (22) has 0 as a simple eigenvalue with corresponding eigenfunction $(\Phi, 0)$. Moreover, all the other eigenvalues are positive and stand apart from 0 . Thus, it follows from perturbation theory [32] that problem (21) has a unique eigenvalue $\mu_{n}$ which is near to zero for large $n$. In particular, all the other eigenvalues of problem (21) have positive real parts and stand apart from 0 . Note that $\mu_{n}$ is simple real eigenvalue which converges to zero and we can denote the corresponding eigenfunction $\left(\xi_{n}, \eta_{n}\right)$ such that

$$
\lim _{n \rightarrow \infty}\left(\xi_{n}, \eta_{n}\right) \longrightarrow(\Phi, 0) .
$$

Now we prove that $\mu_{n}<0$ for large $n$ in following. Multiplying the first equation of (21) with $\Phi$ and integrating on $\Omega$, we get

$$
\begin{aligned}
-\int_{\Omega} & \Delta \xi_{n} \Phi \\
& -\int_{\Omega} \Phi \xi_{n}\left(a_{n}-2 u_{n}-b v_{n} e^{-k u_{n}}+b u_{n} v_{n} m e^{-k u_{n}}\right) \\
& +\int_{\Omega} b u_{n} e^{-k u_{n}} \eta_{n} \Phi=\int_{\Omega} \mu_{n} \xi_{n} \Phi .
\end{aligned}
$$

Meanwhile, multiplying the first equation of (3) with $(u, v)=$ $\left(u_{n}, v_{n}\right)$ by $\xi_{n}$ and integrating, we obtain

$$
-\int_{\Omega} \Delta u_{n} \xi_{n}=\int_{\Omega} u_{n} \xi_{n}\left(a_{n}-u_{n}-b v_{n} e^{-k u_{n}}\right),
$$

Thanks to $u_{n}=s_{n} \Phi+O\left(s^{2}\right)$, adopting variational principle, by the above equation, we have

$$
-\int_{\Omega} \Delta \xi_{n} \Phi=\int_{\Omega} \Phi \xi_{n}\left(a_{n}-u_{n}-b v e^{-k u_{n}}\right)+O\left(s_{n}^{2}\right) .
$$

According to (24) and (26), we have

$$
\begin{aligned}
& -\int_{\Omega} \xi_{n} \Phi u_{n}\left(1-b v_{n} m e^{-k u_{n}}\right)-\int_{\Omega} b u_{n} e^{-k u_{n}} \eta_{n} \Phi \\
& =\int_{\Omega} \Phi \mu \xi_{n}+O\left(s_{n}^{2}\right) .
\end{aligned}
$$

Taking $(u(s), v(s))=\left(s \Phi+O\left(s^{2}\right), \theta_{c}+s \Psi+O\left(s^{2}\right)\right)$ into (27) and so dividing by $s_{n}$ and taking the limit, we obtain

$$
\lim _{n \rightarrow \infty} \frac{\mu_{n}}{s_{n}}=\frac{\int_{\Omega} \Phi^{3}\left(1-b k \theta_{c}\right)}{\int_{\Omega} \Phi^{2}}
$$

recalling that $\int_{\Omega} \Phi^{3}\left(1-b m \theta_{c}\right)<0$, it is easy to see that $\mu<0$ for large $n$. This proves our claim.

Next, in order to prove the existence of at least two positive solutions, we may use reduction to absurdity and

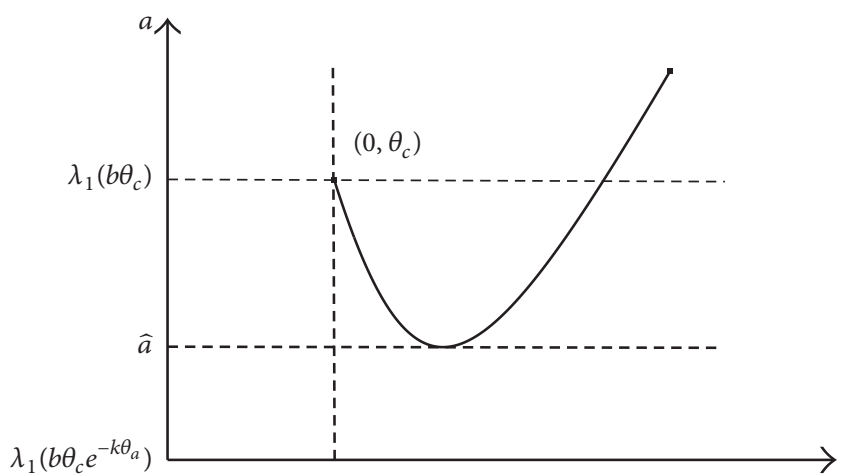

FIGURE 1: Bifurcation sketch map.

suppose that (3) has a unique coexistence state $(\widehat{u}, \widehat{v})$; it follows from local bifurcation theory that solutions must be positive solutions bifurcated from $\left(0, \theta_{c}\right)$ near $\tilde{a}$; moreover, $(\widehat{u}, \widehat{v})$ is nondegenerate and the corresponding linearized eigenvalue problem has a unique eigenvalue $\widehat{\mu}$ such that $\operatorname{Re}(\widehat{\mu})<0$ with multiplicity one. Thanking to the above facts, it is easy to see that $I-F^{\prime}(\widehat{u}, \widehat{v})$ is invertible and does not have property $\alpha$ on $\bar{W}$, and it follows from Lemma 1(ii) that $\operatorname{index}_{w}(F,(\widehat{u}, \widehat{v}))=(-1)^{1}=-1$. Thus, applying Lemmas 4-5 and the additivity property of the index, we obtain

$$
\begin{aligned}
1= & \operatorname{index}_{w}\left(F, D^{\prime}\right) \\
= & \operatorname{index}_{w}(0,0)+\operatorname{index}_{w}\left(F,\left(\theta_{a}, 0\right)\right) \\
& \quad+\operatorname{index}_{w}(F,(\widehat{u}, \widehat{v}))=0+0+1-1=0,
\end{aligned}
$$

which derives a contradiction. The proof is completed.

Remark 12. Theorem 6 gives the multiplicity of positive solutions when $k \geq 0$. Note that since $\int_{\Omega} \Phi^{3}\left(1-b m \theta_{c}\right)<0$ with $a_{1}<0$ for $d \ll 1$, there exists $a=a(s) \in\left(\lambda_{1}, \widetilde{a}\right)$. Remark 10 shows that there is no positive solution of (1) if $a>\lambda_{1}\left(b \theta_{c} e^{-k \theta_{c}}\right)$. Hence, by establishing a bifurcation sketch map as in Figure 1, we can easily get the fact that there must be at least two coexistence states for $a \in(\widehat{a}, \widetilde{a})$ and some $\widehat{a} \in\left(\lambda_{1}\left(b \theta_{c} e^{-k \theta_{c}}\right), \lambda_{1}\left(b \theta_{c}\right)\right)$.

Theorem 13. Suppose that $c>\lambda_{1}, a>\lambda_{1}+b(c+d /(k e))$, and $b(c+d /(k e)) k<1$. If

$$
\Gamma b^{2}+\Gamma(a k+1)^{2} d^{2}+2 a k b d+4 b k\left(c+\frac{d}{(k e)}\right) \leq 4,
$$

where

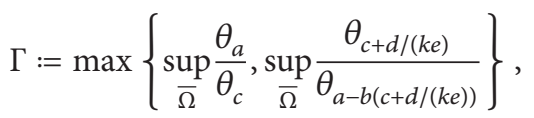

then (3) has a unique positive solution.

Proof. We suppose that $\left(u_{1}, v_{1}\right)$ and $\left(u_{2}, v_{2}\right)$ are two positive solutions of (3). Due to $c>\lambda_{1}$ and $a>\lambda_{1}+b(c+d /(k e))$, 
it follows from the comparison argument for elliptic problem that we have the following results:

$$
\begin{aligned}
\theta_{a-b(c+d /(k e))} & \leq u_{i} \leq \theta_{a} ; \\
\theta_{c} & \leq v_{i} \leq \theta_{c+d /(k e)},
\end{aligned}
$$

$$
i=1,2 .
$$

Setting $A:=u_{1}-u_{2} ; B:=v_{1}-v_{2}$, according to system (3) and the differential mean value theorem, there exists $\xi_{1}, \xi_{2} \in$ $\left[u_{1}, u_{2}\right]$ such that $A$ and $B$ satisfy

$$
\begin{aligned}
& -\Delta A-A\left(a-u_{1}-b v_{1} e^{-k u_{1}}\right)+u_{2} A \\
& -u_{2} b\left(v_{2} k e^{-k \xi_{1}} A-e^{-k u_{1}} B\right)=0, \quad x \in \Omega, \\
& -\Delta B-B\left(c-v_{1}+d u_{1} e^{-k u_{1}}\right)+v_{2} B \\
& -\left(e^{-k u_{2}}-u_{1} k e^{k \xi_{2}}\right) v_{2} d A=0, \quad x \in \Omega,
\end{aligned}
$$$$
A=B=0, \quad x \in \partial \Omega \text {. }
$$

In view of the fact that $\left(u_{1}, v_{1}\right)$ is a solution of (3), 0 is the principal eigenvalue for the following two eigenvalue problems:

$$
\begin{aligned}
-\Delta \chi-\chi\left(a-u_{1}-b v_{1} e^{-k u_{1}}\right) & =0, \\
& x \in \Omega, \quad \chi=0, x \in \partial \Omega, \\
-\Delta \omega-\omega\left(c-v_{1}+d u_{1} e^{-k u_{1}}\right) & =0, \\
& x \in \Omega, \omega=0, x \in \partial \Omega .
\end{aligned}
$$

Hence, applying Rayleigh's formula for the principal eigenvalue, it follows that

$$
\begin{aligned}
& \int_{\Omega}\left[|\nabla A|^{2}-A^{2}\left(a-u_{1}-b v_{1} e^{-k u_{1}}\right)\right] \geq 0, \\
& \int_{\Omega}\left[|\nabla B|^{2}-B^{2}\left(c-v_{1}+d u_{1} e^{-k u_{1}}\right)\right] \geq 0 .
\end{aligned}
$$

Multiplying two equations of (33) by $A$ and $B$, respectively, and integrating on $\Omega$, we get the fact that

$$
\begin{aligned}
& \int_{\Omega}\left[\left(1-v_{2} b k e^{k \xi_{1}}\right) u_{2} A^{2}\right. \\
& +\left(u_{2} b e^{-k u_{1}}+u_{1} v_{2} k d e^{-k \xi_{2}}-v_{2} d e^{-k u_{2}}\right) A B \\
& \left.+v_{2} B^{2}\right] \leq 0 .
\end{aligned}
$$

Thanks to $1-v_{2} b k e^{-k \xi_{1}} \geq 1-b(c+d /(k e)) k>0$, thus

$$
\begin{aligned}
I: & =\left(u_{2} b e^{-k u_{1}}+u_{1} v_{2} k d e^{-k \xi_{2}}-v_{2} d e^{-k u_{2}}\right)^{2}-4(1 \\
& \left.-v_{2} b k e^{-k \xi_{1}}\right) u_{2} v_{2}=\left(u_{2} b e^{-k u_{1}}\right)^{2}+\left(u_{1} k e^{-k \xi_{2}}\right. \\
& \left.-e^{-k u_{2}}\right)^{2} v_{2}^{2} d^{2}+2\left(u_{2} b e^{-k u_{1}}\right)\left(u_{1} k e^{-k \xi_{2}}-e^{-k u_{2}}\right) v_{2} d \\
& -4\left(1-v_{2} b k e^{-k \xi_{1}}\right) u_{2} v_{2} \leq\left(u_{2} b e^{-k u_{1}}\right)^{2}+\left(u_{1} k e^{-k \xi_{2}}\right.
\end{aligned}
$$

$$
\begin{aligned}
& \left.-e^{-k u_{2}}\right)^{2} v_{2}^{2} d^{2}+2 u_{1} u_{2} k b e^{-\left(k u_{1}+k \xi_{2}\right)} v_{2} d \\
& +4 u_{2} v_{2}^{2} b k e^{-k \xi_{1}}-4 u_{2} v_{2}=u_{2} v_{2}\left[\frac{u_{2}}{v_{2}}\left(b e^{-k u_{1}}\right)^{2}\right. \\
& +\left(u_{1} k e^{-k \xi_{2}}+e^{-k u_{2}}\right) \frac{v_{2}}{u_{2}} d^{2}+2 u_{1} k b d e^{-\left(k u_{1}+k \xi_{2}\right)} \\
& \left.+4 v_{2} k b e^{-k \xi_{1}}-4\right] \leq 0
\end{aligned}
$$

Hence, the integral in (36) has a nonnegative value, and then $A, B \equiv 0$ which show the desired results.

\section{Stability and Multiplicity of Positive Solutions}

In this section, the multiplicity, stability, and some uniqueness of positive solutions of (3) are considered by using $k$ as a parameter. Next, some lemmas to obtain the main results of this section are given. These lemmas will show that there exist some upper and lower solutions which do not depend on $k$ and indicate the nondegeneracy at any solution of (3) with certain hypothesis. In the following, an asymptotic result is given firstly.

Lemma 14. Suppose that $a>\lambda_{1}, c>\lambda_{1}$. For any given small $\varepsilon>0$, there exists $K(\varepsilon)$ such that for $k>K(\varepsilon)$, (3) has at least one positive solution $(u, v)$ which satisfies

$$
\begin{gathered}
\theta_{a-\varepsilon} \leq u \leq \theta_{a} \\
\theta_{c} \leq v \leq \theta_{c+\varepsilon}
\end{gathered}
$$

Proof. Set $\underline{U}=(\underline{u}, \underline{v})=\left(\theta_{a-\varepsilon}, \theta_{c}\right), \bar{U}=(\bar{u}, \bar{v})=\left(\theta_{a}, \theta_{a+\varepsilon}\right)$. It is easy to see that functions $u\left(a-u-b v e^{-k u}\right)$ and $v\left(c-v+d u e^{-k u}\right)$ satisfy the Lipschitz continuous in $\{\underline{U}, \bar{U}\}$. If we can show that $\underline{U}$ and $\bar{U}$ are the upper and lower solutions of (3), respectively, then (3) has at least one positive solution $(u, v) \in\{\underline{U}, \bar{U}\}$ with $k>K$. Thanks to Chapter 10 Comparison Theorems and Monotonicity Methods in [30] for nonquasimonotone functions, it suffices to require that the following hold:

$$
\begin{aligned}
\Delta \bar{u}+\bar{u}\left(a-\bar{u}-b v e^{-k \bar{u}}\right) & \leq 0, \quad x \in \Omega, \\
\Delta \bar{v}+\bar{v}\left(c-\bar{u}-d e e^{-k u}\right) & \leq 0, \quad x \in \Omega, \\
\Delta \underline{u}+\underline{u}\left(a-u-b v e^{-k \underline{u}}\right) & \geq 0, \quad x \in \Omega, \\
\Delta \underline{v}+\underline{v}\left(c-\underline{v}-d u e^{-k u}\right) & \geq 0, \quad x \in \Omega, \\
u(x, 0) & =u_{0}(x), \\
v(x, 0) & =v_{0}(x), \quad x \in \Omega .
\end{aligned}
$$


The first and fourth inequalities in (39) are obvious. Next we check the second and third of (21), by the direct calculation as follows:

$$
\begin{aligned}
\Delta \bar{v} & +\bar{v}\left(c-\bar{u}-d u e^{-k u}\right) \\
& =\Delta \bar{v}+\bar{v}(c+\varepsilon+\bar{v})+\bar{v}\left(-\varepsilon+b u e^{-k u}\right) \\
& =\bar{v}\left(-\varepsilon+b u e^{-k u}\right), \\
\Delta \underline{u} & +\underline{u}\left(a-\underline{u}-b v e^{-k \underline{u}}\right) \\
& =\Delta \underline{u}+\underline{u}(a-\varepsilon-\underline{u})+\underline{u}\left(\varepsilon-b v e^{-k u}\right) \\
& =\underline{u}\left(\varepsilon-b v e^{-k u}\right) .
\end{aligned}
$$

Therefore, for $k \gg 1$, the inequalities hold when

$$
\begin{aligned}
& \Delta \bar{v}+\bar{v}\left(c-\bar{v}+d u e^{-k u}\right) \leq 0, \\
& \Delta \underline{u}+\underline{u}\left(a-\underline{u}-b v e^{-k \underline{u}}\right) \geq 0 .
\end{aligned}
$$

Lemma 15. Suppose that $a>\lambda_{1}, c>\lambda_{1}$.

(i) The positive solution of (3) meets (38) and

$$
(u, v) \longrightarrow\left(\theta_{a}, \theta_{c}\right) \quad \text { as } k \longrightarrow \infty .
$$

(ii) There exists sufficiently large $K(\varepsilon)$ such that, for $k>$ $K(\varepsilon)$, the positive solution of (3) which meets (38) is nondegenerate and linearly stable.

Proof. (i) As $k \rightarrow \infty$, it is clear to see that the operator $F(u, v)$ defined in Section 2 converges to the following operator:

$$
\widehat{A}=(-\Delta+p)^{-1}\left(\begin{array}{c}
u(a-u)+P u \\
v(c-v)+P v
\end{array}\right)
$$

therefore, $(u, v) \rightarrow\left(\theta_{a}, \theta_{c}\right)$.

(ii) Suppose that the result does not hold. Then we can obtain $k \rightarrow \infty$; there exists $\mu_{n}$ with $\operatorname{Re}\left(\mu_{n}\right)<0$ and $\left(\xi_{n}, \eta_{n}\right) \neq$ 0 with $\left\|\xi_{n}\right\|_{L^{2}}^{2}+\left\|\eta_{n}\right\|_{L^{2}}^{2}=1$ such that

$$
\begin{aligned}
&- \Delta \xi_{n}-\xi_{n}\left(a-u_{n}-b v_{n} e^{-k_{n} u_{n}}+b u_{n} v_{n} k_{n} e^{-k_{n} u_{n}}\right) \\
&+b u_{n} e^{-k_{n} u_{n}} \eta_{n}=\mu_{n} \xi_{n}, \quad x \in \Omega, \\
&- \Delta \eta_{n}-\xi_{n}\left(d v_{n} e^{-k_{n} u_{n}}-d u_{n} v_{n} k_{n} e^{-k_{n} u_{n}}\right) \\
&-\left(c-2 v_{n}+d u_{n} e^{-k_{n} u_{n}}\right) \eta_{n}=\mu_{n} \eta_{n}, \quad x \in \Omega, \\
& \xi_{n}=\eta_{n}=0, \quad x \in \partial \Omega,
\end{aligned}
$$

where $\left(u_{n}, v_{n}\right)$ is a positive solution of (1) as $k=k_{n}$ which meets (38). By multiplying $\bar{\xi}$ and $\bar{\eta}$ to two equations of (3), respectively, and integrating on $\Omega$, we get

$$
\begin{aligned}
\mu_{n}= & \int_{\Omega}\left|\nabla \xi_{n}\right|^{2} \\
& -\int_{\Omega}\left|\xi_{n}\right|^{2}\left(a-u_{n}-b v_{n} e^{-k_{n} u_{n}}+b u_{n} v_{n} k_{n} e^{-k_{n} u_{n}}\right) \\
& +\int_{\Omega} b u_{n} e^{-k_{n} u_{n}} \eta_{n} \bar{\xi}_{n}+\int_{\Omega}\left|\nabla \eta_{n}\right|^{2} \\
& -\xi_{n} \bar{\eta}_{n}\left(d v_{n} e^{-k_{n} u_{n}}-d u_{n} v_{n} k_{n} e^{-k_{n} u_{n}}\right) \\
& -\int_{\Omega}\left(c-2 v_{n}+d u_{n} e^{-k_{n} u_{n}}\right)\left|\eta_{n}\right|^{2}
\end{aligned}
$$

where $\bar{\xi}_{n}$ and $\bar{\eta}_{n}$ are the complex conjugates of $\xi_{n}$ and $\eta_{n}$, respectively. By the above equation, it is easy to see that $\operatorname{Im}\left(\mu_{n}\right)$ and $\operatorname{Re}\left(\mu_{n}\right)$ are bounded. Then we can suppose that $\mu_{n} \rightarrow \mu$ and $\operatorname{Re}\left(\mu_{n}\right) \leq 0$. Since $\xi_{n}$ and $\eta_{n}$ are also bounded, so let $\eta_{n} \rightarrow \eta, \xi_{n} \rightarrow \xi$. Taking the limit on (44), we get

$$
\begin{aligned}
-\Delta \xi-\xi\left(a-2 \theta_{a}\right) & =\mu \xi, \quad x \in \Omega, \\
-\Delta \eta-\eta\left(c-2 \theta_{c}\right) & =\mu \eta, \quad x \in \Omega, \\
\xi & =\eta=0, \quad x \in \partial \Omega .
\end{aligned}
$$

It follows from (i) that $\mu$ must be real number with $\mu \leq 0$. If $\xi \neq 0$ then $\mu$ is an eigenvalue of the following problem:

$$
-\Delta \xi-\xi\left(a-2 \theta_{a}\right)=\mu \xi, \quad x \in \Omega, \xi=0, x \in \partial \Omega .
$$

Due to $\lambda_{1}\left(-a+2 \theta_{a}\right)>\lambda_{1}\left(-a+\theta_{a}\right)=0,0 \geq \mu \geq \lambda_{1}\left(-a+2 \theta_{a}\right)$, which is a contradiction, so we get $\xi \equiv 0$. Similarly, we also can prove $\eta \equiv 0$, a contradiction to $\left(\xi_{n}, \eta_{n}\right) \neq 0$; the proof is complete.

Next, by the condition $a>\lambda_{1}\left(b \theta_{c}\right)$, one can easily get the fact that $\left(a+\lambda_{1}\left(b \theta_{c}\right)\right) / 2>\lambda_{1}\left(b \theta_{c}\right)$; then there exists a sufficiently small $\sigma>0$ such that $\left(a+\lambda_{1}\left(b \theta_{c}\right)\right) / 2>\lambda_{1}\left(b \theta_{c+\sigma}\right)$, and then we can know that the problem

$$
\begin{aligned}
&-\Delta \omega=\omega\left(\frac{a+\lambda_{1}\left(b \theta_{c}\right)}{2}-b \theta_{c+\sigma}-\omega\right), \\
& x \in \Omega, \omega=0, x \in \partial \Omega
\end{aligned}
$$

has a unique positive solution $\widetilde{\omega}$ by the result of (7).

Lemma 16. Suppose that $c>\lambda_{1}$ and $a>\lambda_{1}\left(b \theta_{c}\right)$. Then, for some $\sigma$, there exists a sufficiently large $K(\sigma)$ such that the following result holds when $K \geq K(\sigma)$.

(i) $u \geq \widetilde{\omega}$; that is, $\widetilde{\omega}$ is a lower solution for $u$.

(ii) A positive solution of (3) converges to $\left(\theta_{a}, \theta_{c}\right)$ as $k \rightarrow$ $\infty$. Meanwhile, the positive solution is nondegenerate and linearly stable. 
Proof. (i) According to Theorem 3 and the comparison argument, we show $v \leq \theta_{c+d /(k e)}$; then there exists a sufficiently large $K(\sigma)$ such that $k \geq K(\sigma)$ with $d /(k e)<\sigma$; hence

$$
\begin{aligned}
-\Delta u & =u\left(a-u-b v e^{-k u}\right) \\
& \geq u\left(\frac{a+\lambda_{1}\left(b \theta_{c}\right)}{2}-b \theta_{c+d /(k e)}-u\right) \\
& \geq u\left(\frac{a+\lambda_{1}\left(b \theta_{c}\right)}{2}-b \theta_{c+\sigma}-u\right) .
\end{aligned}
$$

So the proof is complete.

(ii) As $k \rightarrow \infty, \widetilde{\omega}$ is a lower solution for $u$ which does not depend on $k$; the proof of (ii) is similar to Lemma 15 , so we omit it.

Lemma 17. Suppose that $c \in\left(\lambda_{1}-d /(k e), \lambda_{1}\right)$ and $a>\lambda_{1}+\tau$; here $\tau:=b(c+d /(k e))$. Then there exists a sufficiently large $K$ such that $k>K$; the following claims hold:

(i) $u \geq \theta_{a-\tau}$.

(ii) The positive solution of (3) is nondegenerate and linearly stable.

Proof. (i) Applying the comparison argument for elliptic problem, we obtain $0 \leq v \leq \theta_{c+d /(k e)} \leq c+d /(k e)$, and so as $k$ is large enough we get

$$
\begin{aligned}
-\Delta u & =u\left(a-u-b v e^{-k u}\right) \\
& \geq u\left[a-b\left(c+\frac{d}{(k e)}\right)-u\right],
\end{aligned}
$$

which proves claim (i).

(ii) Let $c=c_{n}$, where $c_{n} \in\left(\lambda_{1}-d /(k e), \lambda_{1}\right)$. Thanks to $u_{n} \geq \theta_{a-\tau_{n}}$, where $\tau_{n}:=b\left(c_{n}+d /\left(k_{n} e\right)\right)$, then $k_{n}\left\|u_{n}\right\|_{\infty} \rightarrow \infty$. Due to $c_{n} \in\left(\lambda_{1}-d /\left(k_{n} e\right), \lambda_{1}\right)$, hence $\left(u_{n}, v_{n}\right) \rightarrow\left(\theta_{a}, 0\right)$. Since $\lambda_{1}-d /\left(k_{n} e\right) \rightarrow \lambda_{1}$, so $c_{n} \rightarrow \lambda_{1}$.

Set $\widehat{v}_{n}=v_{n} /\left\|v_{n}\right\|_{\infty}$; then we get

$$
\begin{aligned}
\Delta \widehat{v}_{n}+\widehat{v}_{n}\left(c_{n}-v_{n}+d u e^{-k_{n} u_{n}}\right) & =0, \\
x & \in \Omega, \widehat{v}_{n}=0, x \in \partial \Omega .
\end{aligned}
$$

According to standard elliptic regularity theory and Sobolev embedding theorems, we may suppose that $\widehat{v}_{n} \rightarrow \widehat{v}>0$. Taking the limit, the above equation satisfies

$$
\begin{aligned}
-\Delta \widehat{v} & =\lambda_{1} \widehat{v}, \quad x \in \Omega ; \\
\widehat{v} & =0, \quad x \in \partial \Omega, \\
\|\widehat{v}\|_{\infty} & =0 .
\end{aligned}
$$

Hence, $\widehat{v}$ must be the principal eigenfunction corresponding to $\lambda_{1}$ denoted by $\Phi_{1}$ and $\left\|\Phi_{1}\right\|_{\infty}=0$.
In the following, we prove that linearized eigenvalue problem (44) has no eigenvalue $\operatorname{Re}\left(\mu_{n}\right) \leq 0$ as $n \gg 1$. Taking $n \rightarrow \infty$, problem (44) converges to the following:

$$
\begin{gathered}
-\Delta \xi-\left(a-2 \theta_{a}\right) \xi, \quad x \in \Omega, \\
-\Delta \eta-\lambda_{1} \eta=\mu \eta, \quad x \in \Omega \\
\xi=\eta=0, \quad x \in \partial \Omega .
\end{gathered}
$$

Obviously, for (53), 0 is a simple eigenvalue with its corresponding eigenfunction $(\xi, \eta)=\left(0, \Phi_{1}\right)$. Applying the method of the proof in Theorem 11, it suffices to prove $\mu_{n}>0$ for $n \gg 1$. Take an eigenfunction $\left(\xi_{n}, \eta_{n}\right)$ such that $\left(\xi_{n}, \eta_{n}\right) \rightarrow$ $\left(0, \Phi_{1}\right)$.

Applying the integral equation on $\Omega$ which can be established by multiplying $v_{n}$ to the second equation of (44) and integrating on $\Omega$, we get

$$
\begin{array}{r}
-\int_{\Omega} \Delta \eta_{n} v_{n}-\int_{\Omega} \xi_{n} v_{n}\left(d v_{n} e^{-k_{n} u_{n}}-d u_{n} v_{n} k_{m} e^{-k_{n} u_{n}}\right) \\
-\int_{\Omega}\left(c-2 v_{n}+d u_{n} e^{-k_{n} u_{n}}\right) \eta_{n} v_{n}=\int_{\Omega} \mu_{n} \eta_{n} v_{n} .
\end{array}
$$

Multiplying $\eta_{n}$ to the second equation of (3) and using variational principle on $\Omega$, we can deduce the following integral equation:

$$
-\int_{\Omega} \Delta \eta_{n} v_{n}=\int_{\Omega} v_{n} \eta_{n}\left(c_{n}-v_{n}+d u_{n} e^{-k_{n} u_{n}}\right)
$$

By substituting $v_{n}=\widehat{v}_{n}\left\|v_{n}\right\|_{\infty}$ into the above two equations and taking the limit, we can get

$$
\operatorname{Lim}_{n \rightarrow \infty} \frac{\mu_{n}}{\left\|v_{n}\right\|_{\infty}}=\frac{\int_{\Omega} \Phi_{1}^{3}}{\int_{\Omega} \Phi_{1}^{2}}>0
$$

which get the desired result.

Theorem 18. (i) If $a \in\left(\lambda_{1}, \lambda_{1}\left(b \theta_{c}\right)\right), c>\lambda_{1}$, then there exists $a$ sufficiently large $K=K(\varepsilon)$ such that (3) has at least two positive solutions for $k>K(\varepsilon)$.

(ii) If $a>\lambda_{1}\left(b \theta_{c}\right), c>\lambda_{1}$, then there exists a sufficiently large $K=K(\sigma)$ such that (3) has a unique positive solution for $k>K(\varepsilon)$.

(iii) If $\lambda_{1}\left(-d \theta_{a} e^{-m \theta_{a}}\right)<c<\lambda_{1}$ and $a>\lambda_{1}+\tau$, where $\tau$ := $b(c+d /(k e))$, then there exists a sufficiently large $K=K(\sigma)$ such that (3) has a unique positive solution for $k>K(\varepsilon)$.

Proof. (i) Suppose the result is false; we can assume that (3) has a unique positive solution $(\widehat{u}, \widehat{v})$; then $(\widehat{u}, \widehat{v})$ must satisfy (38). As $k$ is large enough, by Lemma 14 , it is easy to see that $I-F^{\prime}(\widehat{u}, \widehat{v})$ is invertible and has no property $\alpha$ on $\bar{\omega}(\widehat{u}, \widehat{v})$. Furthermore, $F^{\prime}$ has no real eigenvalue larger than or equal to one. It follows from Lemma 1(ii) that 
$\operatorname{index}_{w}(F,(\widehat{u}, \widehat{v}))=(-1)^{0}=1$. Applying Lemmas 4-5 and the additivity property of the index, we get

$$
\begin{aligned}
1= & \operatorname{index}_{w}\left(F, D^{\prime}\right) \\
= & \operatorname{index}_{w}(F,(0,0))+\operatorname{index}_{w}\left(F,\left(\theta_{a}, 0\right)\right) \\
& \quad+\operatorname{index}_{w}\left(F,\left(0, \theta_{c}\right)\right)+\operatorname{index}_{w}(F,(\widehat{u}, \widehat{v})) \\
= & 0+0+1+1 .
\end{aligned}
$$

Which derive a contradiction, so the result of (i) is established.

(ii) Suppose that (3) has many positive solutions with the hypothesis of (ii), thanks to the compactness of the operator $F$ which has at most finitely many positive fixed points in the given region $D^{\prime}$ which are denoted by

$$
U_{i}=\left(u_{i}, v_{i}\right), \quad i=1,2, \ldots, k .
$$

Similar to the proof of (i), it is easy to see that $\operatorname{index}_{w}\left(F, U_{i}\right)=$ $(-1)^{0}=1, i=1,2, \ldots, k$. Finally, applying Lemmas 16(ii) and 1(ii), along with the additivity property of the index, we get

$$
\begin{aligned}
k= & \sum_{i=1}^{k} \operatorname{index}_{w}\left(F, U_{i}\right) \\
= & \operatorname{index}_{w}\left(F, D^{\prime}\right)+\operatorname{index}_{w}(F,(0,0)) \\
& \quad+\operatorname{index}_{w}\left(F,\left(\theta_{a}, 0\right)\right)+\operatorname{index}_{w}\left(F,\left(0, \theta_{c}\right)\right) \\
= & 1-0-0-0=1 .
\end{aligned}
$$

Thus, the uniqueness is proved.

(iii) By $-d \theta_{a} e^{-k \theta_{a}} \leq d /(k e)$, we can get $c>\lambda_{1}-d /(k e)$. The proof of (iii) is similar to (ii); just the semitrivial solution $\left(0, \theta_{c}\right)$ does not exist in this case. So we omit it.

Theorem 19. If the assumption of Theorem 18(i) holds, then as $n \rightarrow \infty$, either $\left(u_{n}, v_{n}\right) \rightarrow\left(\theta_{a}, \theta_{c}\right)$ or $\left(k_{n} u_{n}, v_{n}\right) \rightarrow\left(U, \theta_{c}\right)$, where $U$ is a positive solution of

$$
-\Delta U=U\left(a-b \theta_{c} e^{-U}\right), \quad x \in \Omega, U=0, x \in \partial \Omega .
$$

Proof. In the event that $k_{n}\left\|u_{n}\right\|_{\infty} \rightarrow \infty$, it is easy to see that $\left(u_{n}, v_{n}\right) \rightarrow\left(\theta_{a}, \theta_{c}\right)$ holds. Hence, we only investigate the case of $k_{n}\left\|u_{n}\right\|_{\infty}<\infty$. Let $k_{n} u_{n}=U_{n}$, it follows that

$$
\begin{aligned}
-\Delta U_{n} & =U_{n}\left(a-\frac{U_{n}}{k_{n}}-b v_{n} e^{-U_{n}}\right), \quad x \in \Omega, \\
-\Delta v_{n} & =v_{n}\left(c-v_{n}+d \frac{U_{n}}{k_{n}} e^{-U_{n}}\right), \quad x \in \Omega \\
U_{n} & =v_{n}=0, \quad x \in \partial \Omega .
\end{aligned}
$$

Taking $n \rightarrow \infty$, the solution $\left(U_{n}, v_{n}\right)$ of the above problem converges to a nonzero solution of the following problem:

$$
\begin{aligned}
-\Delta U & =U\left(a-b v e^{-U}\right), \quad x \in \Omega, \\
-\Delta v & =v(c-v), \quad x \in \Omega \\
U & =v=0, \quad x \in \partial \Omega .
\end{aligned}
$$

Obviously, the result $\left(k_{n} u_{n}, v_{n}\right) \rightarrow\left(U, \theta_{c}\right)$ holds.
Remark 20. By Theorem 19, we can understand that the exact number of positive solutions is dependent on the multiplicity of positive solutions with the same hypothesis in Theorem 18(i).

\section{Conclusion}

The positive solution of a predator-prey model with Dinosaur functional response under Dirichlet boundary conditions is investigated. Firstly, using the comparison principle and fixed point index theory, the sufficient conditions and necessary conditions on coexistence of positive solutions on the predator-prey model with Dinosaur functional response are established. See Theorems 6-8. Secondly, by virtue of bifurcation theory, perturbation theory of eigenvalues, and the fixed point index theory, we establish the bifurcation of positive solutions to (3) and obtain the stability and multiplicity of the positive solution under certain conditions. See Theorem 11. Furthermore, the local uniqueness result is studied when $b$ and $d$ are small. See Theorem 13. Finally, we investigate the multiplicity, uniqueness, and stability of positive solutions when $k>0$ is large. See Lemmas $15-17$ and Theorems 18-19.

\section{Conflicts of Interest}

The authors declare that there are no conflicts of interest regarding the publication of this paper.

\section{Acknowledgments}

The work was partially supported by the National Natural Science Foundation of China (nos. 61102144, 11371230), the Natural Science Basic Research Plan in Shaanxi Province of China (no. 2016JM6041), Shandong Provincial Natural Science Foundation of China (no. ZR2015AQ001), Research Funds for Joint Innovative Center for Safe and Effective Mining Technology and Equipment of Coal Resources by Shandong Province and SDUST (2014TDJH102), and the president of the Xian Technological University Foundation (XAGDXJJ1423).

\section{References}

[1] Y. Du and Y. Lou, "Some uniqueness and exact multiplicity results for a predator-prey model," Transactions of the American Mathematical Society, vol. 349, no. 6, pp. 2443-2475, 1997.

[2] W. Ko and K. Ryu, "Coexistence states of a predator-prey system with non-monotonic functional response," Nonlinear Analysis: Real World Applications, vol. 8, no. 3, pp. 769-786, 2007.

[3] X. Feng and L. Li, "Uniqueness and multiplicity of a preypredator model with predator saturation and competition," Journal of Applied Mathematics, vol. 2012, Article ID 627419, 30 pages, 2012.

[4] M. Wang and Q. Wu, "Positive solutions of a prey-predator model with predator saturation and competition," Journal of Mathematical Analysis and Applications, vol. 345, no. 2, pp. 708718, 2008.

[5] S. Zhang, X. Meng, T. Feng, and T. Zhang, "Dynamics analysis and numerical simulations of a stochastic non-autonomous 
predator-prey system with impulsive effects," Nonlinear Analysis: Hybrid Systems, vol. 26, pp. 19-37, 2017.

[6] J. Blat and K. J. Brown, "Bifurcation of steady-state solutions in predator-prey and competition systems," Proceedings of the Royal Society of Edinburgh. Section A. Mathematics, vol. 97, pp. 21-34, 1984.

[7] H. Cheng, F. Wang, and T. Zhang, "Multi-state dependent impulsive control for holling i predator-prey model," Discrete Dynamics in Nature and Society, vol. 2012, Article ID 181752, 21 pages, 2012.

[8] T. Zhang, X. Meng, Y. Song, and T. Zhang, "A stage-structured predator-prey SI model with disease in the prey and impulsive effects," Mathematical Modelling and Analysis, vol. 18, no. 4, pp. 505-528, 2013.

[9] X.-Z. Meng, S.-N. Zhao, and W.-Y. Zhang, "Adaptive dynamics analysis of a predator-prey model with selective disturbance," Applied Mathematics and Computation, vol. 266, pp. 946-958, 2015.

[10] Z. Bai, X. Dong, and C. Yin, "Existence results for impulsive nonlinear fractional differential equation with mixed boundary conditions," Boundary Value Problems, vol. 1, pp. 63-71, 2016.

[11] Q. Sun and Y. Cui, "Existence results for $(k, n-k)$ conjugate boundary-value problems with integral boundary conditions at resonance with $\operatorname{ker} l=2$," Boundary Value Problems, vol. 1, p. 29, 2017.

[12] Y. Cui, "Uniqueness of solution for boundary value problems for fractional differential equations," Applied Mathematics Letters, vol. 51, pp. 48-54, 2016.

[13] Z. Bai, S. Zhang, S. Sun, and C. Yin, "Monotone iterative method for fractional differential equations," Electronic Journal of Differential Equations, vol. 2016, article 6, pp. 1-8, 2016.

[14] G. Zhu, X. Meng, and L. Chen, "The dynamics of a mutual interference age structured predator-prey model with time delay and impulsive perturbations on predators," Applied Mathematics and Computation, vol. 216, no. 1, pp. 308-316, 2010.

[15] T. Zhang, W. Ma, X. Meng, and T. Zhang, "Periodic solution of a prey-predator model with nonlinear state feedback control," Applied Mathematics and Computation, vol. 266, pp. 95-107, 2015.

[16] T. Zhang, X. Meng, and T. Zhang, "Global analysis for a delayed SIV model with direct and environmental transmissions," The Journal of Applied Analysis and Computation, vol. 6, no. 2, pp. 479-491, 2016.

[17] S. V. Ivlev, "Experimental ecology of the feeding of fishes," The Quarterly Review of Biology, vol. 38, no. 1, pp. 81-82, 1963.

[18] L. Ling and W. M. Wang, "Dynamics of a Ivlev-type predatorprey system with constant rate harvesting," Chaos, Solitons \& Fractals, vol. 41, no. 4, pp. 2139-2153, 2009.

[19] Q. Wang, M. Fan, and K. Wang, "Dynamics of a class of nonautonomous semi-ratio-dependent predator-prey systems with functional responses," Journal of Mathematical Analysis and Applications, vol. 278, no. 2, pp. 443-471, 2003.

[20] Y. Jia, "A sufficient and necessary condition for the existence of positive solutions for a prey-predator system with Ivlev-type functional response," Applied Mathematics Letters, vol. 24, no. 7, pp. 1084-1088, 2011.

[21] X. Zhuo and F. Zhang, "Stability for a new discrete ratiodependent predator-prey system," Qualitative Theory of Dynamical Systems, pp. 1-14, 2017.

[22] J. Sugie, “Two-parameter bifurcation in a predator-prey system of Ivlev type," Journal of Mathematical Analysis and Applications, vol. 217, no. 2, pp. 349-371, 1998.
[23] R. E. Kooij and A. Zegeling, "A predator-prey model with Ivlev's functional response," Journal of Mathematical Analysis and Applications, vol. 198, no. 2, pp. 473-489, 1996.

[24] H. K. Baek, S. D. Kim, and P. Kim, "Permanence and stability of an Ivlev-type predator-prey system with impulsive control strategies," Mathematical and Computer Modelling, vol. 50, no. 9-10, pp. 1385-1393, 2009.

[25] B. Liu, Y. Zhi, and L.-S. Chen, "The dynamics of a predator-prey model with Ivlev's functional response concerning integrated pest management," Acta Mathematicae Applicatae Sinica, vol. 20, no. 1, pp. 133-146, 2004.

[26] H. Wang, "Dispersal permanence of periodic predator-prey model with Ivlev-type functional response and impulsive effects," Applied Mathematical Modelling, vol. 34, no. 12, pp. 3713-3725, 2010.

[27] X. Wang and J. Wei, "Dynamics in a diffusive predatorprey system with strong Allee effect and Ivlev-type functional response," Journal of Mathematical Analysis and Applications, vol. 422, no. 2, pp. 1447-1462, 2015.

[28] W. Wang, L. Zhang, H. Wang, and Z. Li, "Pattern formation of a predator-prey system with Ivlev-type functional response," Ecological Modelling, vol. 221, no. 2, pp. 131-140, 2010.

[29] W. Ruan, W. Feng, and J. A. Goldstein, "On the fixed point index and multiple steady-state solutions of reaction-diffusion systems," Differential and Integral Equations, vol. 8, no. 2, pp. 371-391, 1995.

[30] J. Smoller, Shock Waves and Reaction-Diffusion Equations, vol. 258 of Grundlehren der Mathematischen Wissenschaften [Fundamental Principles of Mathematical Science], Springer, Berlin, Germany, 1983.

[31] P. H. Rabinowitz, "Some global results for nonlinear eigenvalue problems," Journal of Functional Analysis, vol. 7, no. 3, pp. 487513, 1971.

[32] T. Kato, Perturbation Theory for Linear Operators, vol. 132 of Grundlehren der Mathematischen Wissenschaften, Springer, New York, NY, USA, 1966. 


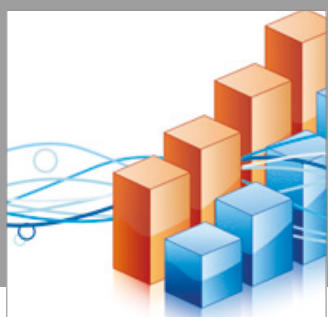

Advances in

Operations Research

vatersals

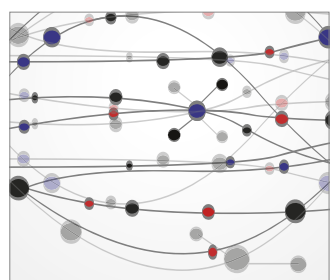

\section{The Scientific} World Journal
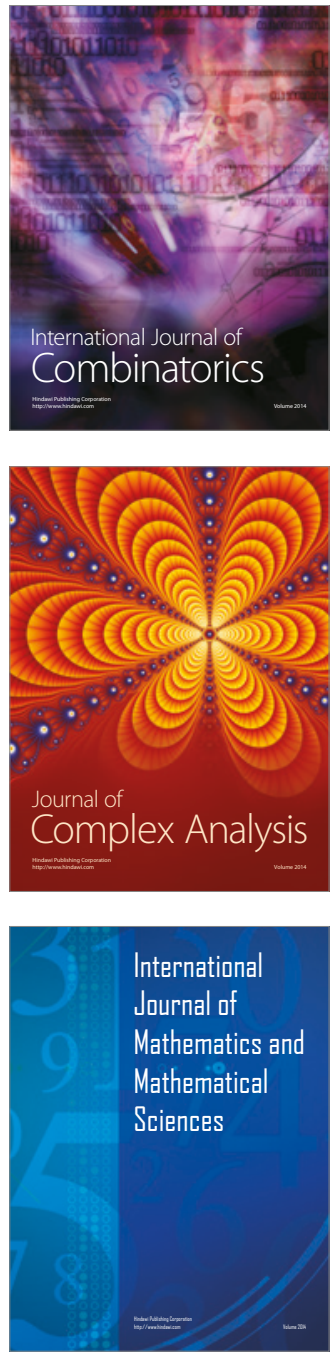
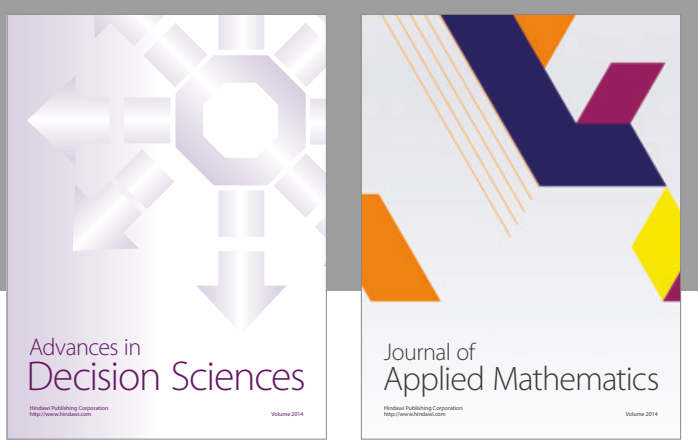

Algebra

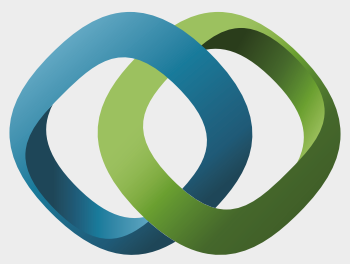

\section{Hindawi}

Submit your manuscripts at

https://www.hindawi.com
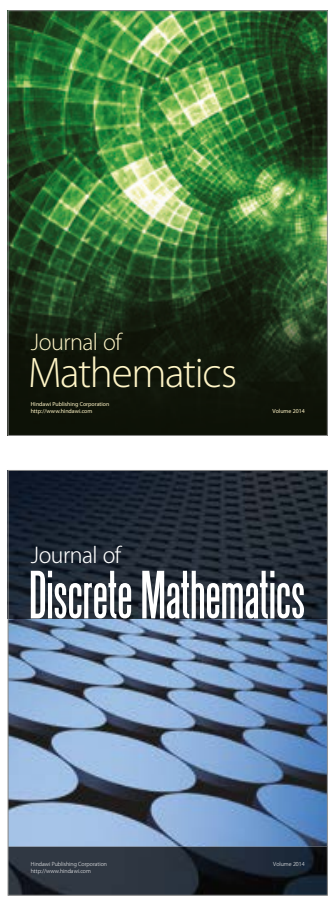

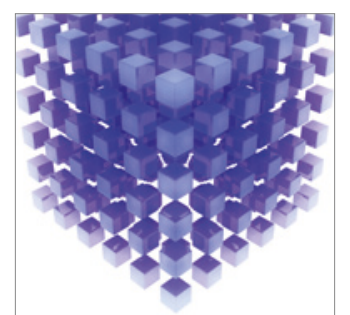

Mathematical Problems in Engineering
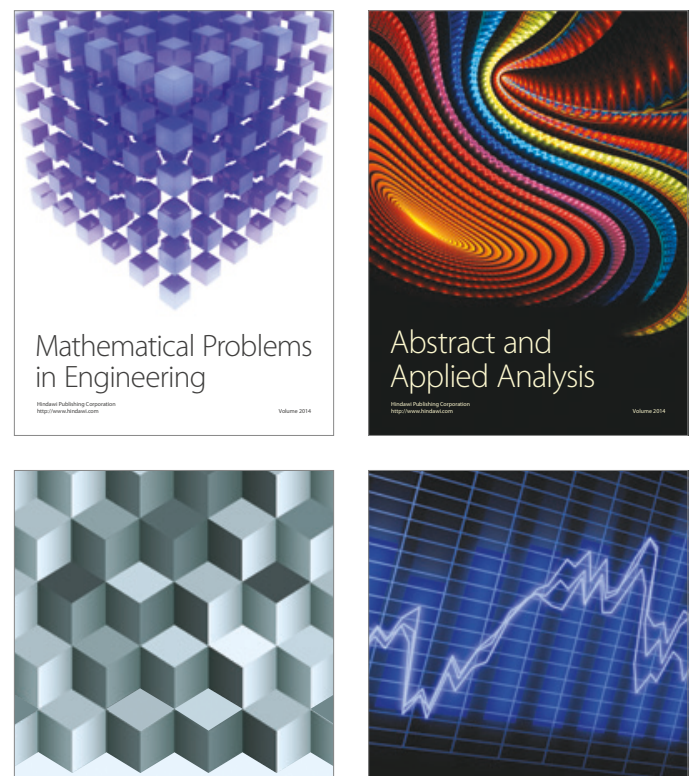

Journal of

Function Spaces

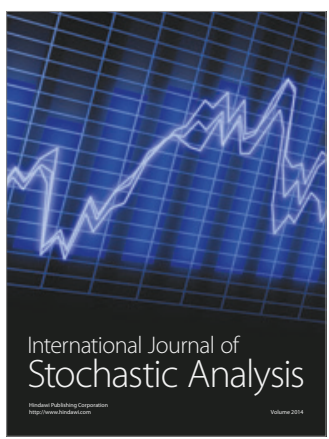

Probability and Statistics
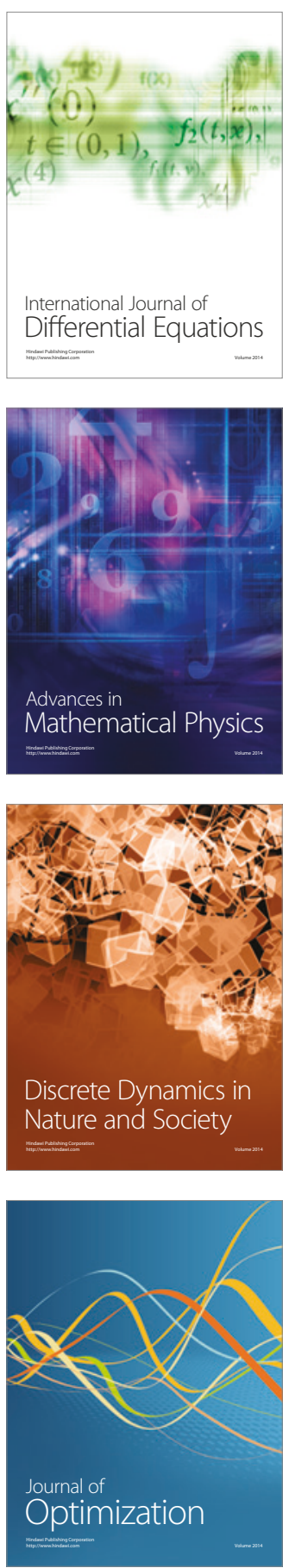